Algebraic $\& \mathcal{G}$ Geometric Topology

Volume 2 (2002) 391-402

Published: 22 May 2002

ATG

\title{
Rational surfaces and symplectic 4-manifolds with one basic class
}

\author{
RonALD Fintushel \\ JONGIL PARK \\ RONALD J. STERN
}

\begin{abstract}
We present constructions of simply connected symplectic 4manifolds which have (up to sign) one basic class and which fill up the geographical region between the half-Noether and Noether lines.
\end{abstract}

AMS Classification 57R57; 57R17

Keywords 4-manifold, Seiberg-Witten invariant

\section{Introduction}

For minimal complex surfaces $S$ of general type, the Noether inequality states that $c_{1}^{2}(S) \geq 2 \chi_{h}(S)-6$, where $\chi_{h}(X)$ denotes the holomorphic Euler number of $X .\left(\chi_{h}(X)=\frac{1}{4}(e(X)+\operatorname{sign}(X))\right.$ where $e$ is the Euler characteristic and sign is the signature of the intersection form.) The line $c_{1}^{2}=2 \chi_{h}-6$ in the $\left(\chi_{h}, c_{1}^{2}\right)$-plane is often called the Noether line. In terms of gauge theory, one of most notable features of a minimal surface of general type is that, up to sign, it has exactly one (Seiberg-Witten) basic class [W]. In [FS1] the first and third authors produced examples of symplectic (see [S]) 4-manifolds with one basic class which lie on the 'half-Noether' line $c_{1}^{2}=\chi_{h}-3$. The inability to construct examples (even smoothly) of 4-manifolds with one basic class and $c_{1}^{2}<\chi_{h}-3$ led them to conjecture that such manifolds fail to exist. Interest in this problem was reignited recently by a paper of Mariño, Moore, and Peradze [MMP] which gave a plausibility argument via physics.

In the current article, we show the existence of symplectic manifolds with one basic class which fill the region in the $\left(\chi_{h}, c_{1}^{2}\right)$-plane between the half-Noether and Noether lines. Specifically we prove:

Theorem 1.1 For every pair of positive integers $(x, c)$ with $0<x-3 \leq c \leq$ $2 x-6$ there is a simply connected symplectic 4-manifold $X$ with $c_{1}^{2}(X)=c$, $\chi(X)=x$ and (up to sign) one basic class. 
We were drawn to this problem by a question of Paul Feehan. The manifolds produced in Theorem 1.1 serve to simplify some of the calculations necessary in the Feehan-Leness program to prove the equivalence of the Seiberg-Witten and Donaldson invariants. Another goal of this paper is to exhibit further techniques for constructing 4-manifolds. In light of this we will present two different constructions for the manifold in question. Each construction brings to light interesting properties of rational and elliptic surfaces.

The key to our constructions is to understand configurations of embedded surfaces in rational surfaces which can be rationally blown down. We close this introduction by reminding the reader of the notion of rational blowdown. (See [FS1] and [P].) Let $C_{n}$ denote the simply connected smooth 4-manifold with boundary obtained by plumbing $n-1$ disk bundles over the 2 -sphere according to the linear diagram:

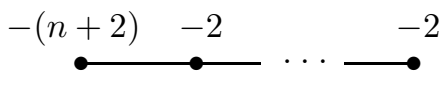

Here, each node denotes a disk bundle over $S^{2}$ with Euler class indicated by the label; an interval indicates that the endpoint disk bundles are plumbed, i.e. identified fiber to base. Label the homology classes represented by the spheres in $C_{n}$ by $S_{0}, S_{1}, \ldots, S_{n-2}$ so that the self-intersections are $S_{0}^{2}=-(n+2)$ and, for $j=1, \ldots, n-2, S_{j}^{2}=-2$. Further, orient the spheres so that $S_{j} \cdot S_{j+1}=+1$. Then $C_{n}$ is a 4-manifold with negative definite intersection form and with boundary the lens space $L\left(n^{2}, 1-n\right)$. The lens space $L\left(n^{2}, 1-n\right)=\partial C_{n}$ bounds a rational ball $B_{n}$ with $\pi_{1}\left(B_{n}\right)=\mathbb{Z}_{n}$ and a surjective inclusion-induced homomorphism $\pi_{1}\left(L\left(n^{2}, 1-n\right)=\mathbb{Z}_{n^{2}} \rightarrow \pi_{1}\left(B_{n}\right)\right.$. If $X$ is a smooth 4-manifold containing an embedded copy of $C_{n}$, its 'rational blowdown' is the result of replacing $C_{n}$ by the rational ball $B_{n}$. Rationally blowing down $C_{n}$ increases $c_{1}^{2}$ by $n-1$ but does not change $\chi_{h}$. It is a theorem of Symington [S] that if the ambient manifold is symplectic, and each sphere $S_{i}$ of a configuration $C_{n}$ is a symplectic submanifold, the resultant manifold of the rational blowdown is also symplectic.

A key result is:

Theorem 1.2 [FS1] Let $X$ be a simply connected smooth 4-manifold with $b^{+}>1$ and containing the configuration $C_{n}$. Suppose that all the (SeibergWitten) basic classes $k$ of $X$ satisfy

$$
k \cdot S_{i}=0, i=1, \ldots, n-2, \text { and }\left|k \cdot S_{0}\right| \leq n .
$$

Then the result of rationally blowing down $C_{n}$ is a smooth 4-manifold whose 
basic classes are in one-to-one correspondence with the basic classes $k$ of $X$ satisfying $\left|k \cdot S_{0}\right|=n$.

The authors gratefully acknowledge the following external support. The first author was partially supported NSF Grant DMS0072212, the second author by KOSEF 981-0103-015-2, and the third author by NSF Grant DMS9971667.

\section{Line arrangements and 4-manifolds}

In this section we shall construct rational surfaces which contain Riemann surfaces of self-intersection 0, along which one is able to form fiber sums. The result of these fiber sums will be elliptic surfaces, Horikawa surfaces, and symplectic manifolds which sit on the "half-Noether line" $c_{1}^{2}=\chi_{h}-3$. There are certainly other constructions of these manifolds (cf. [FS1]) and we shall describe one such in the next section, however the description below is the most convenient for our purposes.

Let $q$ be an integer $\geq 4$. To construct the first rational surface, consider the arrangement of $q$ lines in $\mathbf{C P}^{2}$ formed by taking $q-2$ lines through a common point and two more lines in general position. Blow up the multiple point $x_{0}$ to obtain a configuration of rational curves in $\mathbf{C P} \mathbf{P}^{2} \# \overline{\mathbf{C P}}^{2}$ representing $q H-(q-2) E$, where $H$ denotes the class of a line and $E$ the exceptional curve. Smooth double points to obtain a smooth embedded holomorphic curve of self-intersection $4 q-4$ and genus $q-2$ (as seen via the adjunction formula). Now blow up $4 q-4$ more points along the embedded surface to obtain the rational surface $R(q)$ with $c_{1}^{2}(R(q))=12-4 q$ and with a surface $\Sigma_{R(q)}$ of genus $q-2$ with trivial normal bundle. Furthermore, since an exceptional curve $E_{i},(i=1, \ldots, 4 q-4)$ is a 2 -sphere that intersects $\Sigma_{R(q)}$ in one point, the complement, $R(q) \backslash \Sigma_{R(q)}$ is simply connected.

To construct the second rational surface, start with the arrangement of lines in $\mathbf{C P}^{2}$ obtained by taking $p-3$ lines in $\mathbf{C P}^{2}$ meeting in one point and then adding three more lines in general position. Blow up the point of multiplicity $p-3$ to obtain a configuration of rational curves in $\mathbf{C P}^{2} \# \overline{\mathbf{C P}}^{2}$ representing $p H+$ $(p-3) E$. After smoothing the double points, we obtain a smooth embedded holomorphic curve of self-intersection $6 p-9$ and genus $2 p-5$. Finally, blow up $6 p-9$ points along the embedded surface to obtain the rational surface $S(p)$ with $c_{1}^{2}(S(p))=17-6 p$ and with a surface $\Sigma_{S(p)}$ of genus $2 p-5$ with trivial normal bundle. Note also that $\Sigma_{S(p)}$ intersects all the exceptional classes, and $S(p) \backslash \Sigma_{S(p)}$ is simply connected. 
Define $X_{p}$ to be the symplectic 4-manifold obtained by taking the fiber sum of $R(2 p-3)$ and $S(p)$ along $\Sigma_{R(2 p-3)}$ and $\Sigma_{S(p)}$. (Note that both these surfaces have genus $2 p-5$.) For fiber sums along surfaces of genus $g$, one has the general formulas

$$
\begin{aligned}
& c_{1}^{2}\left(A \#_{\Sigma} B\right)=c_{1}^{2}(A)+c_{1}^{2}(B)+(8 g-8), \\
& \chi_{h}\left(A \#_{\Sigma} B\right)=\chi_{h}(A)+\chi_{h}(B)+(g-1) .
\end{aligned}
$$

It follows that $c_{1}^{2}\left(X_{p}\right)=2 p-7$ and $\chi_{h}\left(X_{p}\right)=2 p-4$; so $c_{1}^{2}\left(X_{p}\right)=\chi_{h}\left(X_{p}\right)-3$. Since the complements of $\Sigma_{R(2 p-3)}$ and $\Sigma_{S(p)}$ in $R(2 p-3)$ and $S(p)$ are simply connected, so is $X_{p}$.

These manifolds, $X_{p}$ all have holomorphic Euler number $\chi_{h}\left(X_{p}\right)$ even. To obtain examples with odd $\chi_{h}$, modify the above construction as follows: Start once more with the arrangement consisting of $p-3$ lines through a single point and 3 further lines in general position. Blow up the multiple point of multiplicity $p-3$ and also one of the double points to obtain a configuration of rational curves in $\mathbf{C P}^{2} \# 2 \overline{\mathbf{C P}}^{2}$ representing the homology class $p H-(p-$ 3) $E-2 E_{1}$. After smoothing the double points of the configuration one obtains a smooth embedded holomorphic curve of self-intersection $6 p-13$ and genus $2 p-6$. Blow up $6 p-13$ points along the embedded surface to obtain the rational surface $S^{\prime}(p)$ with $c_{1}^{2}\left(S^{\prime}(p)\right)=20-6 p$ and with a surface $\Sigma_{S^{\prime}(p)}$ of genus $2 p-6$ which has a trivial normal bundle.

Define $X_{p}^{\prime}$ to be the symplectic 4-manifold obtained by taking the fiber sum of $R(2 p-4)$ and $S^{\prime}(p)$ along the genus $2 p-6$ surfaces $\Sigma_{R(2 p-4)}$ and $\Sigma_{S^{\prime}(p)}$. Then $c_{1}^{2}\left(X_{p}^{\prime}\right)=2 p-8$ and $\chi_{h}\left(X_{p}\right)=2 p-5$; so again, $c_{1}^{2}\left(X_{p}^{\prime}\right)=\chi_{h}\left(X_{p}^{\prime}\right)-3$, and as above, $X_{p}^{\prime}$ is simply connected.

\section{Construction via rational blowdowns}

In order to compute the Seiberg-Witten invariants of the symplectic 4-manifolds $X_{p}$ and $X_{p}^{\prime}$, it is useful to have an alternative construction. We first concentrate on $X_{p}$. Let $R=R(2 p-3)$ and $\Sigma_{R}=\Sigma_{R(2 p-3)}$, and let $S=S(p)$ and $\Sigma_{S}=\Sigma_{S(p)}$. Then $\Sigma_{R}$ represents the homology class

$$
(2 p-3) H-(2 p-5) E-\sum_{i=1}^{8 p-16} E_{i} \in H_{2}\left(\mathbf{C P}^{2} \# \overline{\mathbf{C P}}^{2} \#(8 p-16) \overline{\mathbf{C P}}^{2}\right) .
$$

The rational surface $R$ contains the configuration $C=C_{2 p-6}$ which is a linear plumbing of $2 p-7$ holomorphic spheres: 


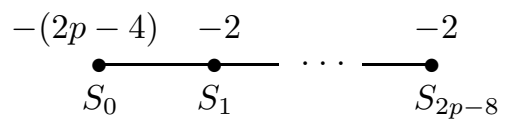

where

$$
S_{0}=H-\sum_{i=1}^{2 p-3} E_{i}, \quad S_{1}=E_{2 p-3}-E_{2 p-2}, \ldots, \quad S_{2 p-8}=E_{4 p-12}-E_{4 p-11} .
$$

Notice that $\Sigma_{R}$ is disjoint from the configuration $C$. This configuration can be rationally blown down by replacing it with a rational ball $B_{2 p-6}$ with $\pi_{1}=$ $\mathbb{Z}_{2 p-6}$. We claim that the rational surface $S$ is the result of rationally blowing down $C$. Since $\Sigma_{R}$ is contained in the complement of $C$, it gives rise to a surface in the new manifold.

Proposition 3.1 Rational blowdown of the configuration $C$ in $R$ yields $S$, and the surface $\Sigma_{R}$ becomes $\Sigma_{S} \subset S$.

Proof We shall prove this by rationally blowing down $C$ together with $6 p-9$ exceptional curves in $R$. The result will be $\mathbf{C P}^{2} \# \overline{\mathbf{C P}}^{2}$, and $\Sigma_{R}$ will get blown down to the class $p h-(p-3) e$, where $h$ and $e$ represent the obvious classes in $\mathbf{C P}^{2} \# \overline{\mathbf{C P}}^{2}$. (We shall use lower case notation in order not to confuse these classes with those used in the description of $\Sigma_{R}$.)

The $6 p-9$ exceptional curves in $R$ to be blown down are $\left\{E_{4 p-10}, \ldots, E_{8 p-16}\right\}$, and $\left\{H-E-E_{1}, \ldots, H-E-E_{2 p-4}\right\}$. These curves are all disjoint from $C$ (and from each other). Thus, if we choose, we may first blow down all the exceptional curves and then rationally blow down the configuration $C$. Blowing down the $E_{j}, j=4 p-10, \ldots, 8 p-16$ we obtain $\mathbf{C P}^{2} \# \overline{\mathbf{C P}}^{2} \#(4 p-9) \overline{\mathbf{C P}}^{2}$ containing the blown down surface $\Sigma_{R}^{\prime}$ which represents the homology class $(2 p-3) H-(2 p-5) E-\sum_{i=1}^{4 p-11} E_{i}$.

Next blow down the exceptional curves $H-E-E_{i}, i=1, \ldots, 2 p-4$. The result is a rational surface $Q$ which has

$$
\left\{\alpha=H-E, \beta=H-\sum_{i=1}^{2 p-4} E_{i}, E_{2 p-3}, \ldots, E_{4 p-11}\right\}
$$

as a basis for $H_{2}(Q)$. (Here we have compacted notation. If we denote the blow down map $R \rightarrow Q$ by $\pi$, then we should write $\alpha=\pi_{*}(H-E)$, etc. This abbreviated notation should not cause any confusion, and we will continue to use it below.) Note that both $\alpha$ and $\beta$ are represented by holomorphic spheres. 
Furthermore, $\alpha$ has self-intersection 0 , and it intersects $\beta$ once, hence for any nonnegative integer $k$, the class $k \alpha+\beta$ is also represented by an embedded 2-sphere. This series of blowdowns takes $\Sigma_{R}^{\prime}$ to a surface $\Sigma_{R}^{\prime \prime}$ which represents the class

$$
(4 p-7) H-(4 p-9) E-2 \sum_{i=1}^{2 p-4} E_{i}-\sum_{j=2 p-3}^{4 p-11} E_{j}=(4 p-9) \alpha+2 \beta-\varepsilon
$$

in $H_{2}(Q)$ (where $\varepsilon=\sum_{j=2 p-3}^{4 p-11} E_{j}$ ).

In $Q$, the sphere $S_{0}$ of the configuration $C$ is given by $S_{0}=\beta-E_{2 p-3}$. The configuration defines a subspace of the second homology whose orthogonal complement $H_{2}(C)^{\perp}$ has basis $\left\{\gamma_{1}, \gamma_{2}\right\}$ where

$$
\gamma_{1}=(2 p-5) \alpha+\beta \text { and } \gamma_{2}=\alpha-\varepsilon
$$

with intersection matrix:

$$
\left(\begin{array}{cc}
2 p-5 & 1 \\
1 & -(2 p-7)
\end{array}\right)
$$

Both $\gamma_{1}$ and $\gamma_{2}$ are represented by embedded holomorphic 2-spheres in $Q \backslash C$. We have already seen this for $\gamma_{1}$, and it is clear for $\gamma_{2}$. Because $H_{2}(C)$ is negative definite, it follows easily that $H_{2}(C)^{\perp}=H_{2}(Q \backslash C)$, and in terms of our generators, $\Sigma_{R}^{\prime \prime}=2 \gamma_{1}+\gamma_{2}$.

Rationally blow down $C$, replacing it with the rational ball $B_{2 p-6}$. The result is a symplectic $([\mathrm{S}])$ 4-manifold $Y=(Q \backslash C) \cup B_{2 p-6}$, and the classes $\gamma_{1}$ and $\gamma_{2}$ rationally generate $H_{2}(Y)$. Since $\gamma_{1}$ is represented by a symplectic 2-sphere of self-intersection $2 p-5>0$, it follows from a theorem of McDuff [M] that $Y$ must be $\mathbf{C P}^{2} \# \overline{\mathbf{C P}}^{2}$.

If we view $Y$ as the ruled surface $\mathbb{F}_{2 p-7}$ with fiber class $f$ and positive and negative section classes $s_{+}$and $s_{-}$, then $\gamma_{1}$ and $\gamma_{2}$ are identified as $\gamma_{1}=s_{+}+f$ and $\gamma_{2}=s_{-}$. Note that this agrees with the model presented in [FS1] where it is shown that $\mathbb{F}_{2 p-7}$ is the union of $B_{2 p-6}$ and a regular neighborhood of spheres representing $s_{+}+f$ and $s_{-}$. Since in $\mathbb{F}_{2 p-7}$ we have

$$
s_{+}+f=(p-2) h-(p-3) e \text { and } s_{-}=(4-p) h+(p-3) e .
$$

It follows that

$$
h=\frac{1}{2}\left(\gamma_{1}+\gamma_{2}\right) \text { and } e=\frac{1}{2 p-6}\left((p-4) \gamma_{1}+(p-2) \gamma_{2}\right) .
$$

In this process, the surface $\Sigma_{R}$ has been blown down to a genus $2 p-5$ surface representing $2 \gamma_{1}+\gamma_{2}=p h+(p-3) e$; so when we blow up $6 p-9$ times, we get $\Sigma_{S}$, and this proves the proposition. 
Similarly, let $R^{\prime}=R(2 p-4), S^{\prime}=S^{\prime}(p)$, and $\Sigma_{R}^{\prime}=\Sigma_{R(2 p-4)}, \Sigma_{S}^{\prime}=\Sigma_{S^{\prime}(p)}$. Then $\Sigma_{R}^{\prime}$ represents the homology class

$$
(2 p-4) H-(2 p-6) E-\sum_{i=1}^{8 p-20} E_{i} \in H_{2}\left(\mathbf{C P}^{2} \# \overline{\mathbf{C P}}^{2} \#(8 p-20) \overline{\mathbf{C P}}^{2}\right)
$$

and $R^{\prime}$ contains the configuration $C^{\prime}=C_{2 p-7}$ composed of

$$
S_{0}^{\prime}=H-\sum_{i=1}^{2 p-4} E_{i}, \quad S_{1}^{\prime}=E_{2 p-4}-E_{2 p-3}, \ldots, \quad S_{2 p-9}^{\prime}=E_{4 p-14}-E_{4 p-13} .
$$

Proposition 3.2 Rational blowdown of the configuration $C^{\prime}$ in $R^{\prime}$ yields $S^{\prime}$ and the surface $\Sigma_{R}^{\prime}$ becomes $\Sigma_{S}^{\prime} \subset S^{\prime}$.

Proof This can be proved in a fashion similar to the proposition above. After blowing down $E_{4 p-12}, \ldots, E_{8 p-20}$ and $H-E-E_{2}, \ldots, H-E-E_{2 p-5}$, all of which are orthogonal to $C^{\prime}$, we are left with $U=\mathbf{C P}^{2} \# \overline{\mathbf{C P}}^{2} \#(2 p-7) \overline{\mathbf{C P}}^{2}$. A basis for $H_{2}(U)$ is given by

$$
\left\{\alpha=H-E, \beta=H-\sum_{i=2}^{2 p-5} E_{i}, E_{1}, E_{2 p-4}, \ldots, E_{4 p-13}\right\},
$$

and $H_{2}\left(C^{\prime}\right)^{\perp}$ is generated by $\left\{\zeta=\alpha-E_{1}, \alpha-\varepsilon,(2 p-7) \alpha+\beta\right\}$, where $\varepsilon=\sum_{2 p-4}^{4 p-13} E_{j}$. The surface $\Sigma_{R}^{\prime}$ gets blown down to a surface $\Sigma_{R, U}^{\prime}$ which, in terms of this basis, represents the class $\zeta+(\alpha-\varepsilon)+2((2 p-7) \alpha+\beta)$.

Rationally blow down $C^{\prime}$ to obtain a simply connected symplectic 4-manifold $W$ with $b^{+}=1, b^{-}=2$, and a symplectically embedded sphere representing $(2 p-7) \alpha+\beta$, a class of square $2 p-7>0$. As above, McDuff's result implies that $W=\mathbf{C P}^{2} \# 2 \overline{\mathbf{C P}}^{2}$. The class $\zeta$ is represented by an exceptional sphere, which we now blow down to obtain a manifold $Y$, which must be diffeomorphic to either $\mathbf{C P}^{2} \# \overline{\mathbf{C P}}^{2}$ or $\mathbf{S}^{2} \times \mathbf{S}^{2}$. The complement of the rational ball $B_{2 p-7}$ in $Y$ has its second homology generated by the classes $(2 p-7) \alpha+\beta+\zeta$ and $\alpha-\varepsilon$ with intersection matrix:

$$
\left(\begin{array}{cc}
2 p-6 & 1 \\
1 & -(2 p-8)
\end{array}\right)
$$

Thus $Y=\mathbb{F}_{2 p-8} \cong \mathbf{S}^{2} \times \mathbf{S}^{2}$ and $s_{+}+f=(2 p-7) \alpha+\beta+\zeta, s_{-}=\alpha-\varepsilon$.

Let $A, B$ denote the classes $\left[S^{2} \times\{\mathrm{pt}\}\right],\left[\{\mathrm{pt}\} \times S^{2}\right]$ in $H_{2}\left(\mathbf{S}^{2} \times \mathbf{S}^{2}\right)$ where the fiber $f=B$. Then if we identify $Y \# \overline{\mathbf{C P}}^{2}=W=\mathbf{C P}^{2} \# 2 \overline{\mathbf{C P}}^{2}$ this identifies: 
$A \leftrightarrow h-e_{1}, B \leftrightarrow h-e$, and $\zeta \leftrightarrow h-e-e_{1}$. Now $\Sigma_{R}^{\prime}$ has been blown down in $W$ to represent

$$
\begin{aligned}
& \zeta+(\alpha-\varepsilon)+2((2 p-7) \alpha+\beta)=2\left(s_{+}+f\right)+s_{-}-\zeta \\
& =2(A+(p-3) B)+(A-(p-4) B)-\zeta=p h-(p-3) e-2 e_{1}
\end{aligned}
$$

which is how $\Sigma_{S}^{\prime}$ is constructed.

The rational surface $R(q+1)=\mathbf{C P}^{2} \# \overline{\mathbf{C P}}^{2} \# 4 q \overline{\mathbf{C P}}^{2}$ may be obtained as the (desingularized) double branched cover of $\mathbf{S}^{2} \times \mathbf{S}^{2}$, branched over two copies of $S^{2} \times\{\mathrm{pt}\}$ and $2 q$ copies of $\{\mathrm{pt}\} \times S^{2}$. In this way we see that $R(q+1)$ admits a 'vertical' genus 0 fibration over $S^{2}$ with fiber class $H-E$ and also a genus $q-1$ 'horizontal' fibration over $S^{2}$.

Lemma 3.3 The fibers of the horizontal fibration of $R(q+1)$ are isotopic to $\Sigma_{R(q+1)}$.

Proof The vertical fibration on $R(q+1)$ has $2 q$ singular fibers, each consisting of an exceptional sphere of multiplicity 2 together with a pair of disjoint spheres of self-intersection -2 , each intersecting the exceptional sphere in a single point. Consider the first such singular fiber - call the spheres, $E_{1}, x$, and $y$. Blowing down $E_{1}$ leaves a pair of exceptional curves, $x+E_{1}$ and $y+E_{1}$. Blow down $E_{2}=x+E_{1}$ to obtain a single sphere $y+E_{1}+E_{2}$ whose square is 0 . This is now the fiber $H-E$ of a genus 0 fibration of $\mathbf{C P}^{2} \# \overline{\mathbf{C P}}^{2} \#(4 q-2) \overline{\mathbf{C P}}^{2}$. It follows that $y=H-E-E_{1}-E_{2}$. In general, the $i$ th singular fiber of the vertical fibration on $R(q+1)$ consists of an exceptional curve $E_{2 i-1}$ of multiplicity 2, along with a pair of disjoint (-2)-spheres, $E_{2 i}-E_{2 i-1}$ and $H-E-E_{2 i-1}-E_{2 i}$. The horizontal fiber $\Lambda$ is homologous to $a H-b E-\sum_{1}^{4 q} c_{i} E_{i}$ for some coefficients $a, b, c_{i}$. Since a generic horizontal fiber intersects a generic vertical fiber in two points, $2=\Lambda \cdot(H-E)=a-b$. Furthermore, a generic horizontal fiber is disjoint from the $(-2)$-spheres which occur as part of the vertical singular fibers. Thus $\Lambda \cdot\left(E_{2 i}-E_{2 i-1}\right)=0=\Lambda \cdot\left(H-E-E_{2 i-1}-E_{2 i}\right)$. The first of these two equalities shows that $c_{2 i-1}=c_{2 i}$ for $i=1, \ldots, 2 q$. The second shows that $a-(a-2)-c_{2 i-1}-c_{2 i}=0$; so $c_{2 i-1}+c_{2 i}=2$, and thus $c_{i}=1$, $i=1, \ldots, 4 q$. Finally, $\Lambda^{2}=0$ gives $a=q+1$. This shows that $\Lambda$ and $\Sigma_{R(q+1)}$ are homologous. Since both are holomorphic curves in $R(q+1)$, they must actually be isotopic (cf. the introduction to [FS3]).

We can now calculate the Seiberg-Witten invariants of $X_{p}$ and $X_{p}^{\prime}$. Let $E(q)$ denote the simply connected elliptic surface with $\chi_{h}=q$ and with no multiple fibers. 
Lemma 3.4 $E(q)$ is diffeomorphic to the fiber sum $R(q+1) \#_{\Sigma_{R(q+1)}} R(q+1)$.

Proof The (desingularized) double cover of $\mathbf{S}^{2} \times \mathbf{S}^{2}$ branched over four copies of $S^{2} \times\{\mathrm{pt}\}$ and $2 q$ copies of $\{\mathrm{pt}\} \times S^{2}$ is $E(q)$. The previous lemma shows that this is the fiber sum as advertised.

It follows from this lemma and Proposition 3.1 that $X_{p}$ is the rational blowdown of a configuration $C_{2 p-6}$ in $E(2 p-4)$. The elliptic fiber $T$ of

$$
E(2 p-4)=R(2 p-3) \#_{\Sigma_{R(2 p-3)}} R(2 p-3)
$$

is obtained from a genus zero fiber on each side, since these spheres intersect $\Sigma_{R(2 p-3)}$ in two points. The genus 0 fiber in $R(2 p-3)$ represents the class $H-E$, and the lead sphere $S_{0}$ of $C_{2 p-6}$ represents $H-\sum_{1}^{2 p-3} E_{i}$.

The basic classes of $E(2 p-4)$ are $\pm 2 j T, j=0, \ldots, p-3$. Of these, only $\pm(2 p-6) T$ intersects $S_{0}$ maximally (with intersection number $\pm(2 p-6)$ ). It follows from Theorem 1.2 that the rational blowdown $X_{p}$ has (up to sign) just one basic class. A similar argument shows that the same is true for $X_{p}^{\prime}$.

Proposition 3.5 The simply connected symplectic manifolds $X_{p}$ and $X_{p}^{\prime}$ $(p \geq 4)$ have (up to sign) one basic class and satisfy $c_{1}^{2}=\chi_{h}-3$.

\section{Construction 1}

In order to fill in the region, $\chi_{h}-3 \leq c_{1}^{2} \leq 2 \chi_{h}-6$ we shall next exhibit symplectic spheres of self-intersection -4 in $X(p)$ and $X^{\prime}(p)$ which can be rationally blown down. These spheres will be built from 'pieces' which intersect $\Sigma_{R(2 p-3)}$ and $\Sigma_{S(p)}$ transversely. In $R(2 p-3)$ there are the exceptional spheres $E_{i}, i=1,2, \ldots, 8 p-16$ which intersect $\Sigma_{R(2 p-3)}$ transversely in a single point. Also, consider a line in $\mathbf{C P}^{2}$ that does not belong to the arrangement for $R(2 p-3)$, but which goes through the singular point of order $2 p-5$ in the arrangement. This line gets blown up to a sphere of self-intersection 0 which intersects $\Sigma_{R(2 p-3)}$ transversely in two points. This sphere represents the class $H-E$. We may form arbitrarily many such disjoint spheres. Denote them by $A_{j}$.

To construct spheres in $S(p)$, recall how it is constructed. The initial arrangement consists of $p-3$ lines through a common point $x_{0}$, and three further lines $L_{i}, i=1, \ldots, 3$, in general position. One then blows up at $x_{0}$ and at further 
points $x_{j}, j=1, \ldots, 6 p-9$ on the arrangement. We can suppose the $x_{2 k-1}$ lie on $L_{1}$ and that $x_{2 k}$ lie on $L_{2}$ for $k=1, \ldots, 3 p-5$ and are arranged so that each pair of points $\left\{x_{2 k-1}, x_{2 k}\right\}$ lies on a line $B_{k}^{\prime}$ through $x_{0}$. After all the blowups, one obtains spheres $B_{k}(k=1, \ldots, 3 p-5)$ of self-intersection -2 in $S(p)$. These spheres intersect $\Sigma_{S(p)}$ transversely in one point (the point of intersection of $B_{k}^{\prime}$ with $\left.L_{3}\right)$. Note that $B_{k}$ is homologous to $H-E-E_{2 k-1}-E_{2 k}$.

Also, there are spheres $C_{\ell}$ with self-intersection 0 that intersect $\Sigma_{S(p)}$ in three points that are obtained from a line in $\mathbf{C P}^{2}$ that goes through the singular point of order $p-3$. The spheres $C_{\ell}$ are homologous to $H-E$.

In $X_{p}$ each of the spheres $E_{i}, A_{j}, B_{k}$, and $C_{\ell}$ is punctured. One can form the fiber sum so that the punctures match up in such a way that $B_{1} \cup A_{1} \cup C_{1} \cup E_{1} \cup E_{2}$ is a symplectic sphere of self-intersection -4 in $X_{p}$. Further, we can arrange so that there are $3 p-5$ disjoint symplectic $(-4)$-spheres constructed in this way. Rationally blowing these down, one at a time, we obtain simply connected symplectic manifolds $X(p, k)$ which have, up to sign one basic class, and with $\chi_{h}(X(p, k))=\chi_{h}\left(X_{p}\right)=2 p-4$, and $c_{1}^{2}(X(p, k))=c_{1}^{2}\left(X_{p}\right)+k=2 p-7+k$, i.e. filling up the region $\chi_{h}-3 \leq c_{1}^{2} \leq \frac{5}{2} \chi_{h}-2, \chi_{h}$ even.

The same construction applied to $X_{p}^{\prime}$ yields the odd $\chi_{h}$ examples. In this case one can construct $3 p-7$ of the spheres $B_{k}$ and thence $3 p-7$ spheres of self-intersection -4 to rationally blow down. We get manifolds $X^{\prime}(p, k)$ with $\chi_{h}\left(X^{\prime}(p, k)\right)=\chi_{h}\left(X_{p}\right)=2 p-5$, and $c_{1}^{2}(X(p, k))=c_{1}^{2}\left(X_{p}^{\prime}\right)+k=2 p-8+k$. So we fill the region $\chi_{h}-3 \leq c_{1}^{2} \leq \frac{5}{2} \chi_{h}-2, \chi_{h}$ odd.

Theorem 4.1 For every pair of positive integers $(x, c)$ with $0<x-3 \leq c \leq$ $\frac{5}{2} x-2$ there is a simply connected symplectic 4-manifold $X$ with $c_{1}^{2}(X)=\bar{c}$, $\chi(X)=x$ and (up to sign) one basic class.

This implies Theorem 1.1.

\section{Construction 2}

We shall now give a second proof of Theorem 4.1 with a construction starting directly with the elliptic surfaces $E(n)$. Fix a pair of positive integers $(x, c)$ with $0<x-3 \leq c \leq \frac{5}{2} x-2$ as in the statement of the theorem, and consider the elliptic surface $E(x)$. It admits an elliptic fibration with $6 x$ cusp fibers and no other singular fibers. Furthermore, $E(x)$ contains, as a symplectic codimension 
0 submanifold, the canonical resolution of the $(2,2 x-1,4 x-3)$ Brieskorn singularity. This contains the configuration of symplectic spheres:

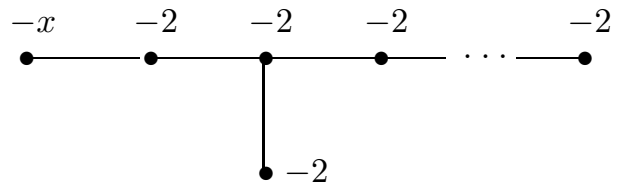

where the linear plumbing to the right of the central node has $4 x-4$ spheres of self-intersection -2 , and where the sphere $S$ of self-intersection $-x$ is a section of the elliptic fibration on $E(x)$ (cf. [FS4]).

Each fiber of the elliptic fibration meets $S$ in a single positive intersection. In particular, consider one of the $6 x$ cusp fibers. If we blow up at the cuspidal point, then in $E(x) \# \overline{\mathbf{C P}}^{2}$ we obtain an embedded sphere representing $f-2 E$ where $f$ is the class of an elliptic fiber in $E(x)$ and $E$ is the class of the exceptional curve. Thus $f-2 E$ represents an embedded sphere that meets $S$ once, positively, in $E(x) \# \overline{\mathbf{C P}}^{2}$. Now symplectically resolve the intersection to obtain a sphere $S^{\prime}$ of self-intersection $-(x+2)$. Comparing with the plumbing diagram above, we see a symplectic embedding of the configuration $C_{x}$ in $E(x) \# \overline{\mathbf{C P}}^{2}$. This process can be repeated until we either exhaust all $6 x$ of the cusp fibers or all $4 x-2$ of the (-2)-spheres across the top of the plumbing.

If we blow up $k$ of the cuspidal points, we obtain a sphere of self-intersection $-(x+2 k)$. This is the lead sphere of the configuration $C_{x+2 k-2}$ which has $x+2 k-4(-2)$-spheres. Thus we can find a symplectic configuration $C_{x+2 k-2}$ in $E(x) \# k \overline{\mathbf{C P}}^{2}$ for $x+2 k-4 \leq 4 x-2$; i.e. for $0 \leq k \leq \frac{3}{2} x+1$. (The $k=0$ case is $C_{x-2} \subset E(x)$.)

In $E(x) \# k \overline{\mathbf{C P}}^{2}$, rationally blow down the configuration $C_{x+2 k-2}$. This yields a symplectic manifold with $\chi_{h}=x$ and $c_{1}^{2}=x+k-3$. Applying the blowup formula [FS2] shows that the Seiberg-Witten basic classes of $E(x) \# k \overline{\mathbf{C P}}^{2}$ have the form

$$
\beta\left(m ; \varepsilon_{1}, \ldots, \varepsilon_{k}\right)=m f+\varepsilon_{1} E_{1}+\cdots+\varepsilon_{k} E_{k}
$$

for $|m| \leq x-2, m \equiv x(\bmod 2)$, and $\varepsilon_{i}= \pm 1$ for $1 \leq i \leq k$. We now apply Theorem 1.2. Each of the (-2)-spheres $S_{i}, i=1, \ldots, x+2 k-4$, is embedded in $E(x)$ and is symplectic. Therefore, it follows from the adjunction formula that

$$
0=K_{E(x)} \cdot S_{i}=(x-2) f \cdot S_{i}
$$

where $K_{E(x)}$ denotes the canonical class of $E(x)$. Hence $f \cdot S_{i}=0$ for $i=$ $1, \ldots, x+2 k-4$. Since clearly each $E_{j} \cdot S_{i}=0$, we have

$$
\beta\left(m ; \varepsilon_{1}, \ldots, \varepsilon_{k}\right) \cdot S_{i}=0, \quad i=1, \ldots, x+2 k-4 .
$$


The lead sphere $S_{0}$ of our configuration $C_{x-2 k-2}$ is given homologically by

$$
S_{0}=S-2 E_{1}-\cdots-2 E_{k} .
$$

Hence $\quad \beta\left(m ; \varepsilon_{1}, \ldots, \varepsilon_{k}\right) \cdot S_{0}=m+2 \sum_{i=1}^{k} \varepsilon_{i} \leq m+2 k \leq x+2 k-2$.

Thus the hypotheses of Theorem 1.2 are satisfied. It is now easy to see that only the basic classes $\pm \beta(x-2 ; 1, \ldots, 1)$ satisfy $\beta\left(m ; \varepsilon_{1}, \ldots, \varepsilon_{k}\right) \cdot S_{0}=x+2 k-2$; and so, up to sign, our manifold has just one basic class.

Since this construction yields 4-manifolds with $\chi_{h}=x$ and $c_{1}^{2}=x+k-3$ for $0 \leq k \leq \frac{3}{2} x+1$, the existence of these manifolds again proves Theorem 4.1.

The authors do not know if the families of manifolds produced by our two constructions actually coincide. This is quite plausible and seems to be an interesting question.

\section{References}

[FS1] R. Fintushel and R. Stern, Rational blowdowns of smooth 4-manifolds, Jour. Diff. Geom. 46 (1997), 181-235.

[FS2] R. Fintushel and R. Stern, Immersed spheres in 4-manifolds and the immersed Thom conjecture, Turkish J. Math. 19 (1995), 145-157.

[FS3] R. Fintushel and R. Stern, Symplectic surfaces in a fixed homology class, J. Diff. Geom., 52 (2000), 203-222.

[FS4] R. Fintushel and R. Stern, The canonical class of a symplectic 4-manifold, Turkish J. Math. 25 (2001), 137-145.

[MMP] M. Mariño, G. Moore, and G. Peradze, Superconformal invariance and the geography of four-manifolds, Comm. Math. Phys. 205 (1999), 691-735.

[M] D. McDuff, The structure of rational and ruled symplectic 4-manifolds, Jour. Amer. Math. Soc. 3 (1990), 679-712.

[P] J. Park, Seiberg-Witten invariants of generalised rational blow-downs, Bull. Austral. Math. Soc. 56 (1997), 363-384.

[S] M. Symington, Symplectic rational blowdowns, Jour. Diff. Geom. 50 (1998), 505-518.

[W] E. Witten, Monopoles and four-manifolds, Math. Res. Lett. 1 (1994), 769-796.

Department of Math, Michigan State University, East Lansing, MI 48824, USA

Department of Math, Konkuk University, 1 Hwayang-dong

Kwangjin-gu, Seoul 143-701, Korea

Department of Math, University of California, Irvine, CA 92697, USA

Email: ronfint@math.msu.edu, jipark@konkuk.ac.kr, rstern@math.uci.edu

Received: 19 February 2002

Algebraic 83 Geometric Topology, Volume 2 (2002) 